\title{
Ground segmentation algorithm of lidar point cloud based on Ray-Ransac
}

\author{
Yawei Zhao, Yanju Liu*, Yang Yu, Jiawei Zhou \\ Shenyang Ligong University, No. 6, Nanping Middle Road, Hunnan District, \\ Shenyang, 110159 \\ China
}

Received: March 4, 2021. Revised: July 27, 2021. Accepted: August 10, 2021. Published: August 12, 2021.

\begin{abstract}
Aiming at the problems of poor segmentation effect, low efficiency and poor robustness of the Ransac ground segmentation algorithm, this paper proposes a radar segmentation algorithm based on RayRansac. This algorithm combines the structural characteristics of three-dimensional lidar and uses ray segmentation to generate the original seed point set. The random sampling of Ransac algorithm is limited to the original seed point set, which reduces the probability that Ransac algorithm extracts outliers and reduces the calculation. The Ransac algorithm is used to modify the ground model parameters so that the algorithm can adapt to the undulating roads. The standard deviation of the distance from the point to the plane model is used as the distance threshold, and the allowable error range of the actual point cloud data is considered to effectively eliminate the abnormal points and error points. The algorithm was tested on the simulation platform and the test vehicle. The experimental results show that the lidar point cloud ground segmentation algorithm proposed in this paper takes an average of $\mathbf{5 . 7 8 4}$ milliseconds per frame, which has fast speed and good precision. It can adapt to uneven road surface and has high robustness.
\end{abstract}

Keywords - Ground segmentation, lidar, Ray-Ransac algorithm, point cloud.

\section{INTRODUCTION}

In driverless technology, the use of lidar to construct a point cloud map is an important part. It is an important basis for completing high-precision maps, path planning, and other advanced intelligent tasks in the later stage. How to remove the interference from the ground and other obstacles during the composition process has become one of the research hotspots of scholars at home and abroad.

Yang et al., Yang et al., and Zhang et al. converted the original point cloud data into two-dimensional feature images and separated the road surface using traditional image segmentation methods [1-3]. In the process of point cloud conversion, this method loses the information of point cloud height and reduces the accuracy of ground separation.

Montemerlo et al. used the distance between threedimensional data rings to determine whether a single threedimensional point belongs to the ground area [4]. Byun et al., Zhu and Liu used Markov random field to complete the ground segmentation [5-6]. Kammel and Pitzer proposed a ground detection method based on the maximum elevation difference of point clouds in the grid. If the maximum elevation difference of the grid is less than a certain threshold, the grid will be marked as the ground area [7]. Although the grid-based detection method is relatively stable, the detection accuracy of this method depends on the grid size. Compared with the original $3 \mathrm{~cm}$ point cloud 0.2 $\mathrm{cm}$ data accuracy, the accuracy of the grid algorithm is lower. Moosmann et al. performed ground segmentation based on the unevenness of the measured points in cylindrical coordinates [8]. Himmelsbach et al. rasterized the three-dimensional data in the form of polar coordinates and performed nonparametric ground fitting for each sector. Ground points and non ground points were separated by calculating the distance between a single point and the fitted line [9]. The Random Sample Consensus (Ransac) ground segmentation algorithm obtains the fitting plane parameters by iterative method and uses the distance from the point to the fitted plane as the segmentation index to distinguish ground points and non-ground points [10-11]. The algorithm is very sensitive to the distance threshold. Unreasonable setting of the distance threshold will lead to over-segmentation and under-segmentation. The traditional sample selection method of Ransac ground segmentation algorithm is random sampling in the overall sample containing a large number of external points. A large number of external points lead to the increase of algorithm iteration times, long algorithm time and low efficiency [12].

Aiming at the problems of poor segmentation effect, low efficiency and poor robustness of the Ransac ground segmentation algorithm, this paper proposes a ground segmentation of lidar point cloud based on Ray-Ransac algorithms. This algorithm uses the ray segmentation method 
to extract the original set of ground seed points (near-ground points or suspected ground points), and then uses the Ransac algorithm to complete the fitting and parameter modification of the ground model [13-17]. The standard deviation of the distance from the point to the plane model is used as the distance threshold. Whether the orthogonal projection distance from the seed point set to the revised ground model is less than the set distance threshold is judged. If it is less than the threshold, the point is classified as a ground point, otherwise it is a non-ground point to complete the ground point cloud segmentation.

\section{AlgORITHM PRINCIPLE OF RAY-RANSAC}

Ransac algorithm is a method of estimating high-precision mathematical model parameters from data containing a large number of external points by iterative methods. It has the characteristics of high robustness and easy implementation [18-19]. The Ransac algorithm has a basic assumption, that is, the sample data contains both internal point data that conforms to the model description and external point or outlier point data that are far from the normal range and cannot adapt to the mathematical model [20].

In the Ransac algorithm, the sample selection method is to randomly sample the overall sample containing a large number of outliers. The random sampling method will inevitably bring uncertainty to the sampling result. The existence of a large number of outliers will increase the number of algorithm iterations, take too long time and inefficient. Therefore, when applying this algorithm to the ground segmentation of Lidar point cloud, this paper improves the selection of the Ransac algorithm's sampling samples, and limits the samples randomly sampled by the Ransac algorithm to the original seed point set generated by the ray method.

The flowchart of the Ray-Ransac algorithm proposed in this paper is shown in Fig. 1.

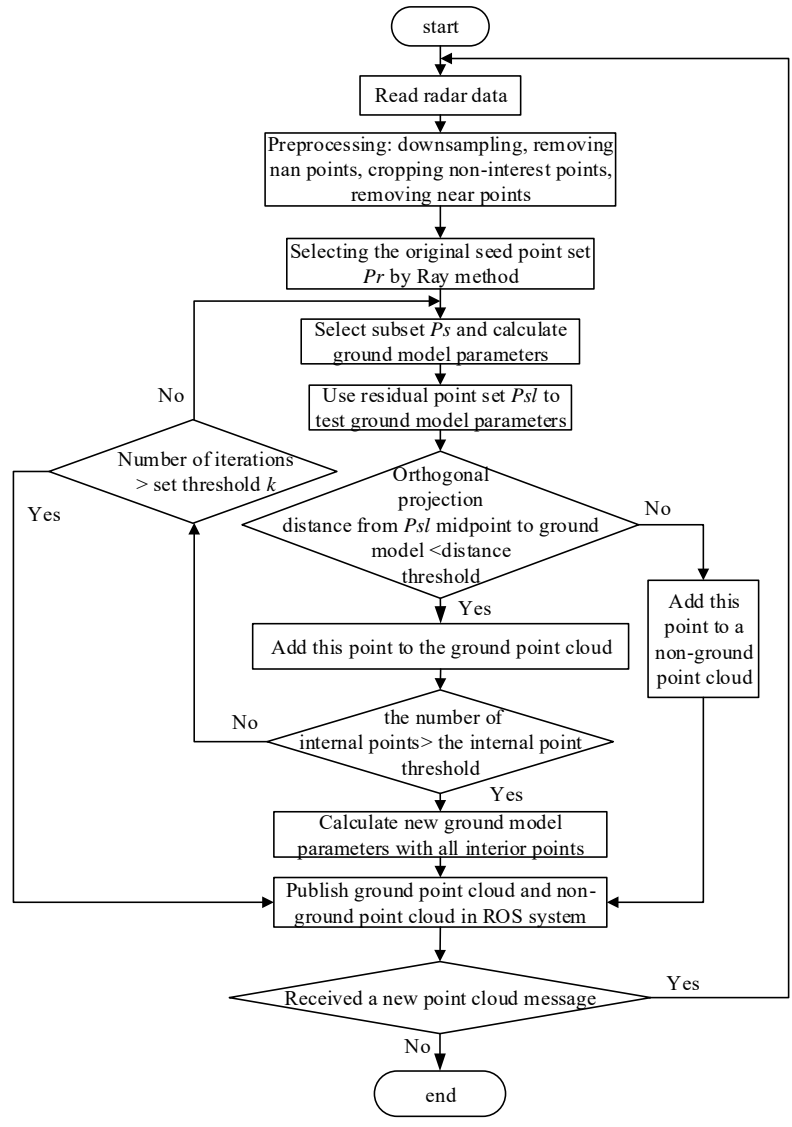

Fig. 1 flowchart of the Ray-Ransac

\section{A. Preprocessing}

(1) Downsampling

In order to reduce the amount of data processed by the point cloud in the later period, the original point cloud data output by the lidar was down-sampled. This article uses the voxel grid filter method (Voxel Grid Filter) to downsample the original point cloud [21]. The size of the downsampled voxel grid is set to $5 \mathrm{~cm}$, that is, the Voxel Grid Filter divides the lidar input point cloud with a small cube with a size of $5 \mathrm{~cm} \times 5 \mathrm{~cm} \times 5 \mathrm{~cm}$. The shape of the small cube is used to represent all the points of the cube, and these points are retained as the output of downsampling.

(2) Clipping and near-point filtering of Lidar point cloud

In the process of segmenting the ground, it is necessary to pay attention to the ground points and near-ground points. For the data above the point of interest, it can be cropped and filtered to reduce the calculation amount of later point cloud data processing. The actual installation of the lidar on the vehicle in this paper is shown in Fig. 2. The lidar installation height is $68 \mathrm{~cm}$, and the point clouds above 50 $\mathrm{cm}$ are cut out. At the same time, the invalid points of the lidar output are eliminated. In order to avoid interference from lidar data, such as reflections on the car, the data smaller than the body radius of $35 \mathrm{~cm}$ is removed in this paper. The near-point data range is shown by the white triangle mark in Fig. 2. 


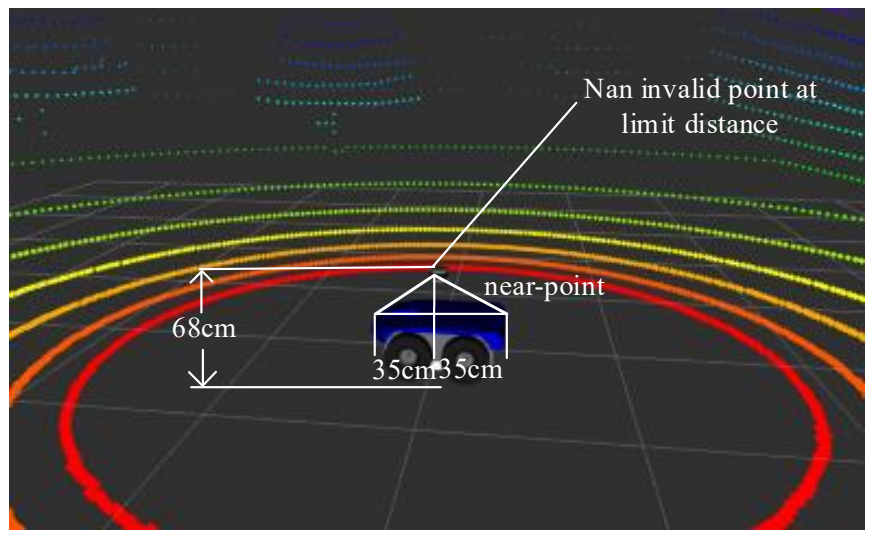

Fig. 2 lidar installation on unmanned vehicles

The four steps of downsampling, eliminating invalid points, cropping non-interest points, and removing near points are all pre-processing steps of the ground segmentation algorithm. Lidar original point cloud and preprocessed point cloud are shown in Fig. 3 and Fig. 4, respectively.

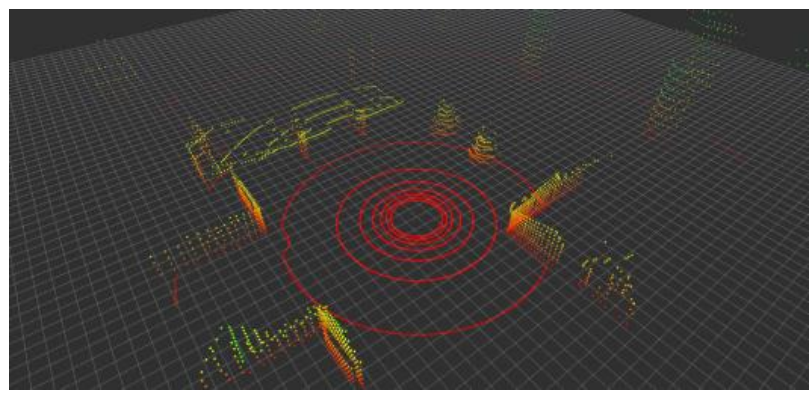

Fig. 3 original point cloud

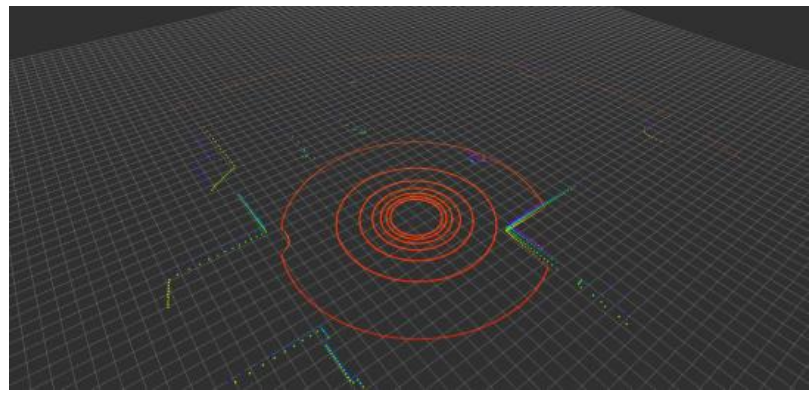

Fig. 4 pre-processed point cloud

\section{B. Ray method to extract the original seed point set}

Three-dimensional lidar has the characteristics of fixed angle and corresponding line number of laser emission. This paper uses this characteristic to design a new method of selecting the original seed point set-ray method. In this paper, the lidar uses VLP-16 lidar. The internal line numbers and angular distributions are shown in Table I. The lidar scan lines with even line numbers are extracted as rays.
Table I. VLP-16 lidar line number and angle distribution

\begin{tabular}{cc}
\hline Line number & angle \\
\hline 0 & $-15^{\circ}$ \\
1 & $1^{\circ}$ \\
2 & $-13^{\circ}$ \\
3 & $3^{\circ}$ \\
4 & $-11^{\circ}$ \\
5 & $5^{\circ}$ \\
6 & $-9^{\circ}$ \\
7 & $6^{\circ}$ \\
8 & $-7^{\circ}$ \\
9 & $9^{\circ}$ \\
10 & $-5^{\circ}$ \\
11 & $11^{\circ}$ \\
12 & $-3^{\circ}$ \\
13 & $13^{\circ}$ \\
14 & $-1^{\circ}$ \\
15 & $15^{\circ}$ \\
\hline
\end{tabular}

The points on the ray are sorted according to the radius distance, and the longitudinal section of two adjacent rings generated is shown in Fig. 5.

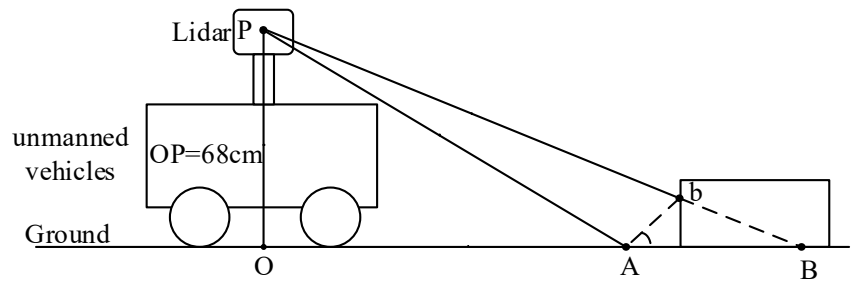

Fig. 5 schematic diagram of longitudinal section of two adjacent rays of a lidar

$\mathrm{OB}$ is the ground; OP is the installation height of the lidar from the ground; PA and PB are adjacent rays emitted by the lidar. When there are no obstacles on the ground, the $b$ point generated by the lidar $\mathrm{PB}$ rays will coincide with the $\mathrm{B}$ point, and $\angle \mathrm{bAB}$ is equal to $0^{\circ}$; When there are obstacles on the ground or the lidar scans to the edge of the road, the PB rays will land on Point $\mathrm{b}$, and $\angle \mathrm{bAB}$ is not equal to $0^{\circ}$. Based on this, it can be determined whether the point scanned by the lidar is near-ground or non-close-ground by judging whether the size of $\angle \mathrm{bAB}$ is within the angle threshold range. Nonnear-ground points are excluded, and the remaining points are used as the original seed point set $P_{r}$.

\section{Ransac algorithm for ground separation}

The core idea of Ransac algorithm is randomness and hypothesis. Randomness is the random selection of sampling data based on the probability of correct data appearing. Randomness simulation can approximate the correct result. Hypothesis is to assume that the selected sampling data are all correct data, then these correct data are used to calculate the corresponding model, and the remaining data are used to 
evaluate the model. In this paper, the steps of using Ransac algorithm to separate the ground are as following:

(1) Randomly extract subsets

This paper mainly focuses on the ground segmentation problem. The ground is a simple plane. The description plane needs at least 3 points. Therefore, a subset $P_{s}$ of 3 points is randomly selected from the original seed point set $P_{r}$.

(2) Build parametric models and solve

The description of the ground model composed of the subset $P_{s}$ is shown in (1):

$$
a x+b y+c z+d=0
$$

$p_{1}\left(x_{1}, y_{1}, z_{1}\right), p_{2}\left(x_{2}, y_{2}, z_{2}\right)$ and $p_{3}\left(x_{3}, y_{3}, z_{3}\right)$ are let to be three points in the subset $P_{s}$. The normal vector of the plane formed by $p_{1}, p_{2}, p_{3}$ is:

$$
\begin{aligned}
& \vec{n}=\overrightarrow{p_{1} p_{2}} \times \overrightarrow{p_{2} p_{3}} \\
& =\left|\begin{array}{ccc}
i & j & k \\
x_{2}-x_{1} & y_{2}-y_{1} & z_{2}-z_{1} \\
x_{3}-x_{1} & y_{3}-y_{1} & z_{3}-z_{1}
\end{array}\right| \\
& =a i+b j+c k
\end{aligned}
$$

Formula (2) is calculated. The determinant is expanded. The point equation of the plane model is solved. Then the point equation is expanded. The equivalent coefficient method is used to solve the ground model parameters a, b, c, and d.

(3) Model checking and parameter updating

After obtaining the initial ground model parameters, the point cloud $P_{s l}$ remaining in the original seed point set is used to test the model and complete the update of the model parameters. The test method is to calculate the distance $d_{p}$ from the point in the point cloud $P_{s l}$ to the orthogonal projection of the ground model. The standard deviation of the distance from the point to the plane model is used as the distance threshold $d_{f}$. Whether the projection distance $d_{p}$ is less than the set plane distance threshold $d_{f}$ is judged. If $d_{p}<d_{f}$, the point belongs to the ground. Otherwise, the point is marked as a non-ground point to complete the ground point cloud segmentation. The distance threshold $d_{f}$ takes into account the allowable error range of the actual point cloud data and can effectively eliminate abnormal points and error points. Finally, the number of ground points under the ground model is counted, that is, the number of internal points of the Ransac algorithm. If the number of internal points is greater than the set threshold, all internal points are used to recalculate the ground model parameters. If it is less than the set threshold, steps (1) to (3) are repeated. If the number of repetitions is greater than the set iteration threshold $k$, the calculation of model parameters is stopped.

(4) Calculation of iteration number $\mathrm{k}$

$p$ is let to be the probability that $n$ points in the original seed point set $P_{r}$ are all interior points, and $w$ is let to be the probability that the internal point is randomly selected in the original seed point set $P_{r}$ each time. $w$ is expressed as:

$$
w=\frac{\text { Inner points of the original seed point set }}{\text { the original seed point set }}
$$

Assuming that $w^{n}$ represents the probability of selecting the inner point each time in $n$ random selections, $1-w^{n}$ represents the probability of selecting the outer point at least once in $n$ random selections. The model established in this case is considered to be an error model, so the probability of an error in $\mathrm{k}$ iterations can be expressed as:

$$
p_{k}=\left(1-w^{n}\right)^{k}
$$

Formula (4) is equal to $1-p$ to obtain (5):

$$
1-p=\left(1-w^{n}\right)^{k}
$$

Logarithms on both sides to get (6):

$$
k=\frac{\log (1-p)}{\log \left(1-w^{n}\right)}
$$

\section{AlgORIthm SimUlation AND ReSUlts}

The algorithm proposed in this paper was first tested on a simulation platform. The main parameters of the hardware platform configuration are a 4-core $\mathrm{CPU}$, a frequency of 2.6 $\mathrm{GHz}$, a graphics card of NVIDIA GEFORCE $720 \mathrm{M}$, and a memory of $8 \mathrm{~GB}$. First, a simulation environment based on Gazebo is set up, as shown in Fig. 6. The blue is the test vehicle model, and a VLP-16 lidar is mounted on it.

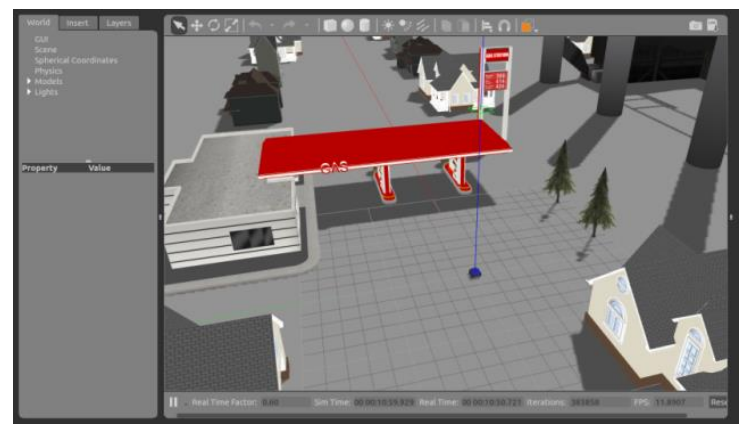

Fig. 6 gazebo simulation environment

After the simulation environment is established, the raw data of the lidar can be seen in the visualization window of rviz as shown in Fig. 7. Fig. 8 is a schematic diagram of ground segmentation using the original Ransac algorithm to fit the plane. The white points in the figure represent the nonground point cloud $P_{n}$ and the purple points represent the ground point cloud $P_{g}$. The point cloud image after the ground segmentation using the Ray-Ransac algorithm is shown in Fig. 9. White represents the non-ground point cloud, and red represents the ground point cloud. 


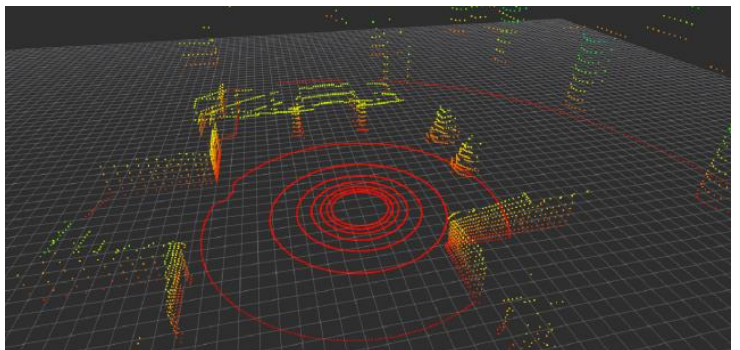

Fig. 7 lidar raw data map

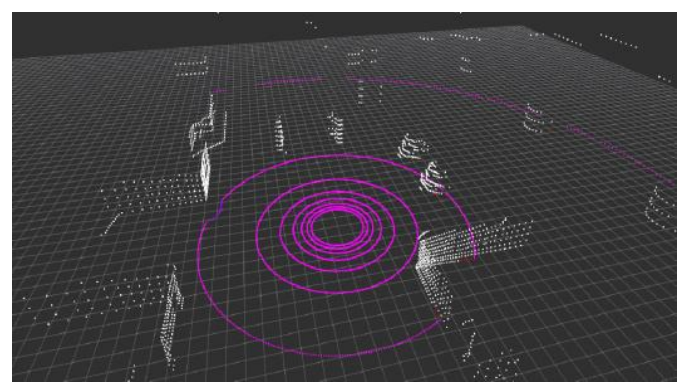

Fig. 8 point cloud image after Ransac segmentation

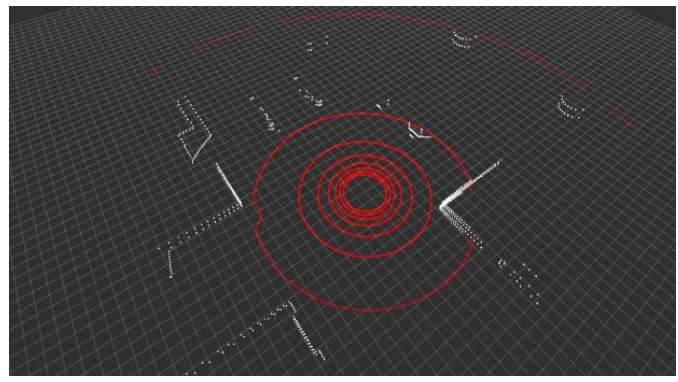

Fig. 9 point cloud image after Ray-Ransac segmentation

The pure ground segmented by Ransac and Ray-Ransac algorithm is shown in Fig. 10. In the figure, white is the ground segmented by Ransac algorithm, and purple is the ground segmented by Ray-Ransac. It can be seen from the figure that at the near point, the two algorithms have the same segmentation effect. However, at the far point, Ransac algorithm recognizes the bottom of the house as the ground, resulting in wrong segmentation. Because Ray-Ransac algorithm uses ray segmentation method to generate the original seed point set, the random sampling samples of Ransac algorithm are only limited to the original seed point set, which reduces the probability of extracting outliers by Ransac algorithm. In addition, Ray-Ransac uses the standard deviation of the distance from the point to the plane model as the distance threshold and considers the allowable error range of the actual point cloud data, which can effectively eliminate abnormal points and error points. Therefore, the Ray-Ransac algorithm can well separate ground and non-ground obstacles at far points.

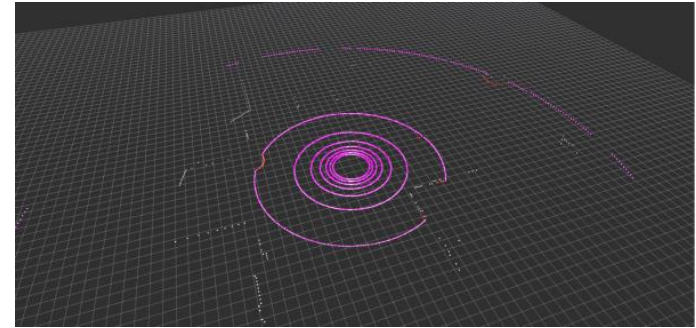

Fig. 10 schematic ground segmentation by two algorithms

Fig. 11 shows the simulation time of ground segmentation by Ransac and Ray-Ransac. After calculation, the average time for ground segmentation using Ransac algorithm is 6.746 milliseconds, and the average time for ground segmentation using Ray-Ransac algorithm is 2.194 milliseconds, which is $66.1 \%$ less than the average time for ground segmentation using Ransac algorithm.

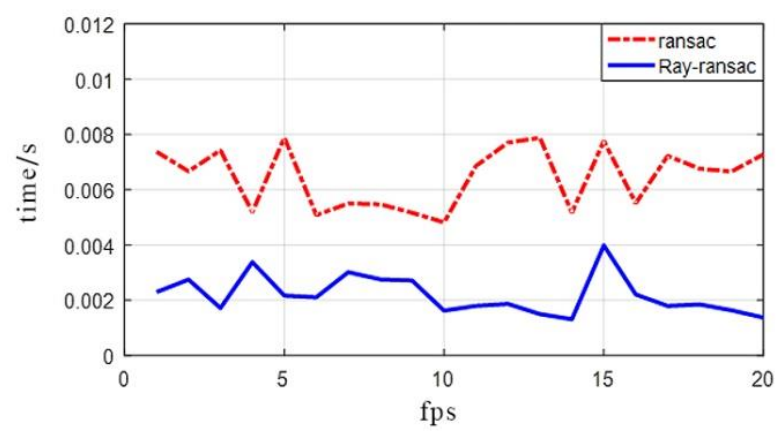

Fig. 11 algorithm simulation takes time

\section{ACTUAL ROAD TEST AND RESULtS}

The environment during the actual road test is more complicated, and the collected 3D point clouds are no longer regular concentric circles. At this time, the ground segmentation results are prone to problems such as excessive segmentation or insufficient segmentation [22]. The computer configuration of the road test is the same as that of the simulation environment. The unmanned vehicle used is shown in Fig. 12, and the raw data image of the lidar is shown in Fig. 13.

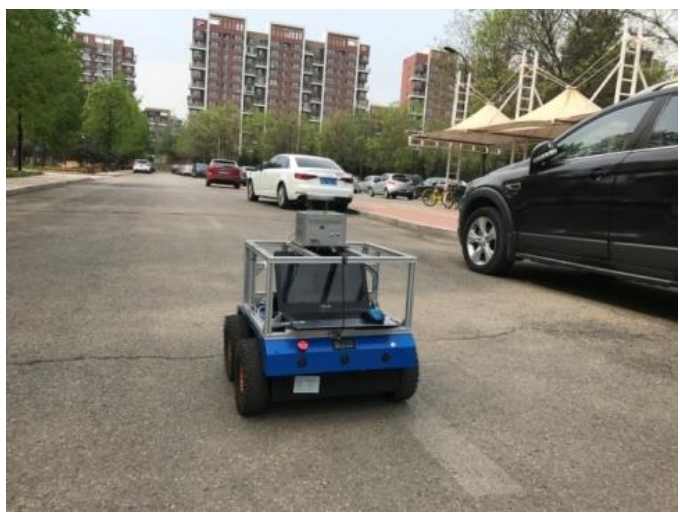


Fig. 12 road-tested unmanned vehicles

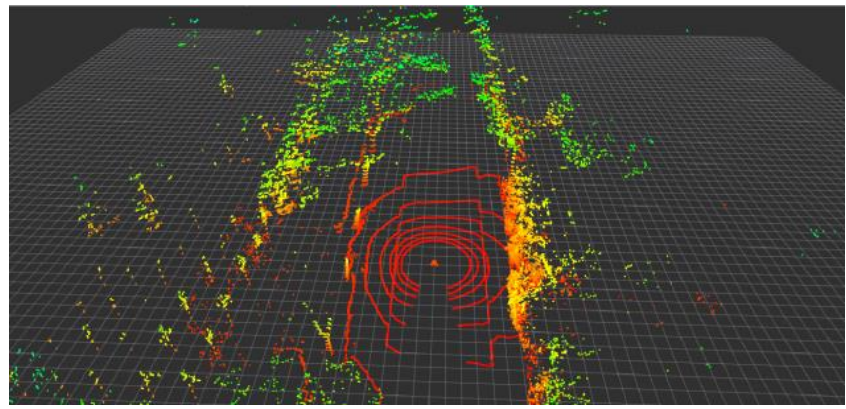

Fig. 13 the raw data image of the lidar point cloud

The measured ground separation results of Ransac and RayRansac algorithms are shown in Fig. 14 and Fig. 15. It can be seen from Fig. 14 that when Ransac was used to segment the ground during road test, the ground was separated from nonground points at a distant point. At the same time, a large number of point clouds on the sidewalk were also divided into the ground, as shown in the blue box mark in Fig. 14. Since the Ray-Ransac algorithm uses the ray segmentation method to generate the original seed point set, and takes the standard deviation of the distance from the point to the plane model as the distance threshold, this method can restrict the Ransac algorithm random sampling sample to the original seed point set, reducing the probability of the Ransac algorithm extracting external points, taking into account the allowable error range of the actual point cloud data, and can effectively eliminate abnormal points and error points. When the Ray-Ransac algorithm is used, the phenomenon of erroneous separation of far points is eliminated, and the point cloud of erroneous separation on the sidewalk is also greatly reduced.

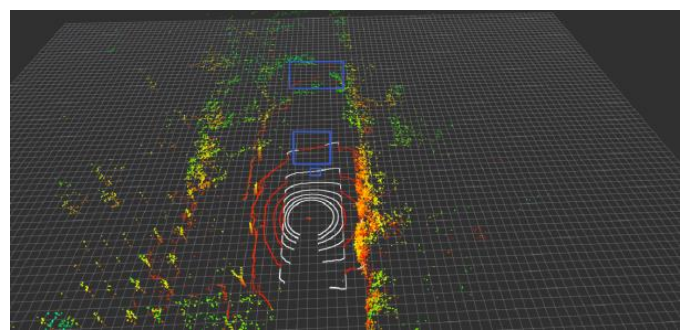

(a) Ransac ground segmentation map

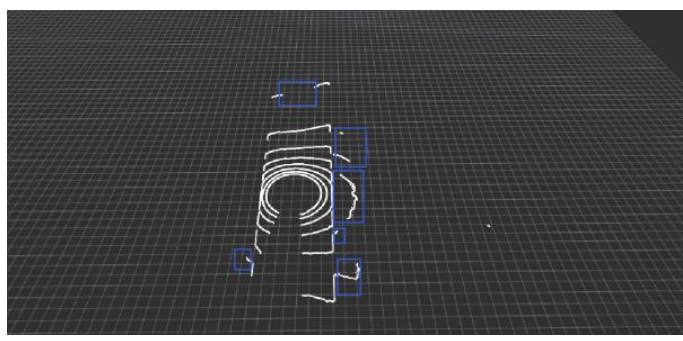

(b) ground after Ransac algorithm

Fig. 14 ground separation results after Ransac road-tested

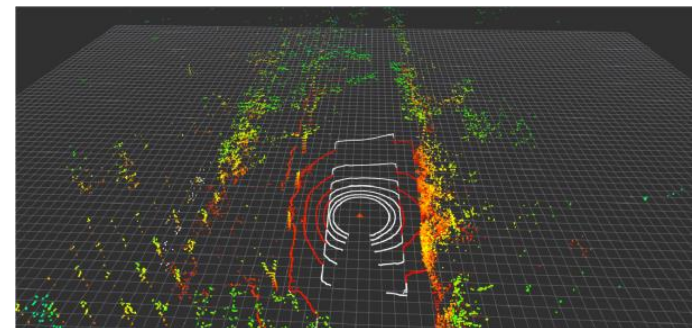

(a) ray-Ransac ground segmentation map

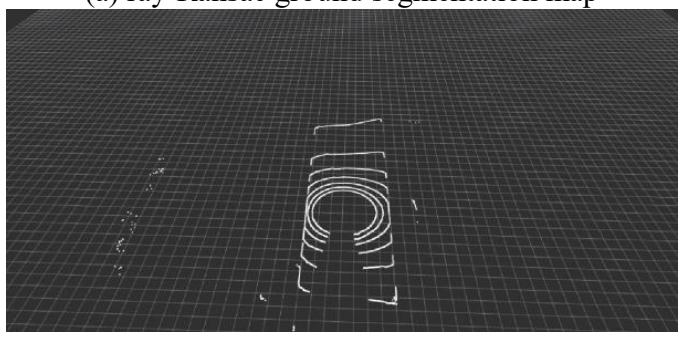

(b) ground after Ray-Ransac algorithm

Fig. 15 ground separation results after Ray-Ransac road-tested

When the unmanned vehicle encounters the deceleration zone as shown in Fig. 16, the Ray-Ransac algorithm can still separate the ground better, and the separation result is shown in Fig. 17.

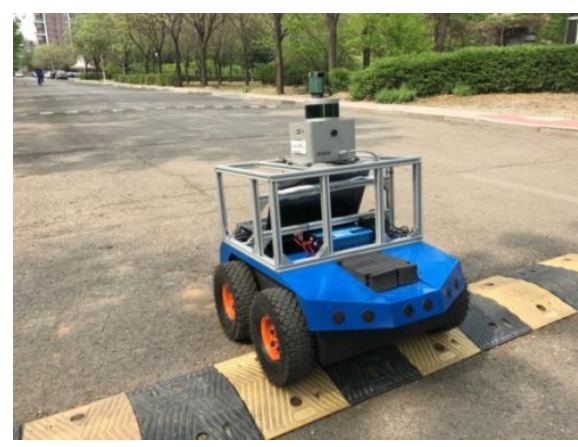

Fig. 16 unmanned vehicle passes speed bump

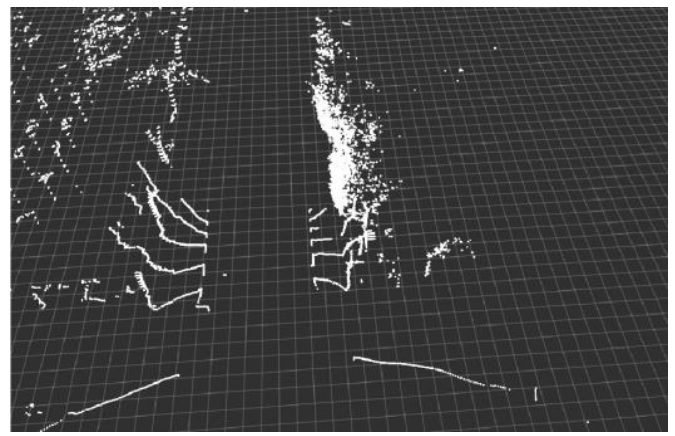

Fig. 17 results of ground separation when passes speed bump

From the observation of the actual road separation results, it can be known that the ground segmentation algorithm proposed in this paper can accurately segment the ground when it encounters uneven roads such as deceleration zones. 
The time consumption of the algorithm during road actual test is shown in Fig. 18. It can be found from the figure that the average time consumption of the algorithm during road actual test is increased compared with the simulation time, and the average time is about 2.87 times of the simulation time. The reason for the increase in time consumption is that when the actual road test is performed, the data of the lidar is no longer the simulation data during the simulation, but the actual threedimensional point cloud data of the lidar collected in real time. The Ray-Ransac algorithm completes the ground segmentation in the industrial computer according to the collected real-time three-dimensional point cloud of the lidar. Therefore, the algorithm time consuming of the road test is affected by the communication rate of the laser radar device. However, the average time taken to segment the ground using the RayRansac algorithm is still much lower than the average time taken using the Ransac algorithm, with the average time reduced from 18.463 milliseconds to 5.784 milliseconds, which is $68.7 \%$ less than the average time for ground segmentation using Ransac algorithm.

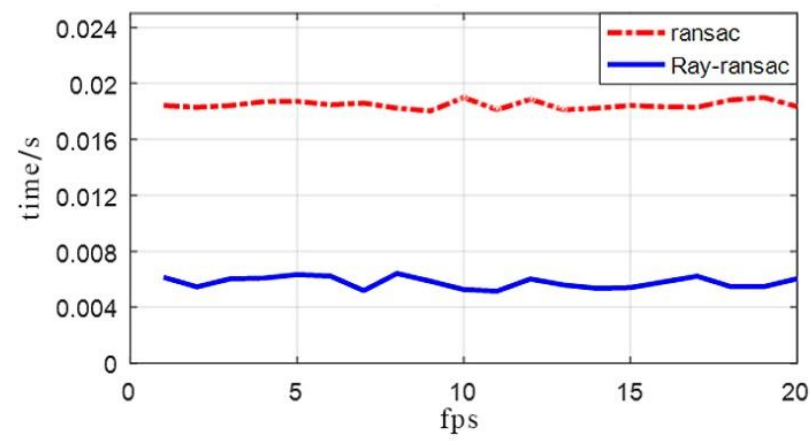

Fig. 18 time consumption of Ray-Ransac algorithm for road test

\section{CONCLUSIONS}

Ground segmentation is a key step in environmental perception, and its performance will directly affect the subsequent autonomous obstacle avoidance and path planning of unmanned vehicles.

This paper proposes a lidar ground segmentation algorithm based on Ray-Ransac. The algorithm first preprocesses the lidar point cloud, then uses the ray method to select the original seed point set. The random sampling samples of Ransac algorithm are limited to the original seed point set, which reduces the probability of Ransac algorithm extracting external points. The Ransac algorithm is used to modify the ground model parameters and completes the discrimination between ground points and non-ground points. The standard deviation of the distance from the point to the plane model is used as the distance threshold, and the allowable error range of the actual point cloud data is considered to effectively eliminate the abnormal points and error points. After the ground segmentation is completed, the final point cloud segmentation results are published in the ROS system.
The simulation and road test results show that compared with the Ransac ground segmentation algorithm, using the Ray-Ransac ground segmentation algorithm proposed in this paper, the ground segmentation of lidar point cloud has fast speed and good accuracy. At the same time, the algorithm can also adapt to uneven road surfaces and has high robustness. Based on the ground segmentation, unmanned vehicles can complete autonomous obstacle avoidance and path planning, which provides an important guarantee for the precise control and safe operation of unmanned vehicles.

Although the algorithm in this paper can adapt to uneven roads, it needs to be further improved due to the need to iteratively fit multiple planes when facing complex ground with large undulations. In the future, the prior information of the radar laser point cloud will be used to guide data sampling and iteratively estimate the ground model quickly, optimize the algorithm flow, and further improve the calculation efficiency.

\section{ACKNOWLEDGMENT}

The study is funded by the National Key Research and Development Program of the Ministry of Science and Technology, Grant No. 2017YFC082100; Liaoning Natural Science Foundation Guidance Program in 2019, Grant No. 2019-ZD-0252; Scientific Research Funding Project of Liaoning Provincial Department of Education, Grant No. LG202009.

\section{References}

[1] B. Yang, Z. Wei, Q. Li, and Q. Mao, "A classificationoriented method of feature image generation for vehicleborne laser scanning points,". Acta Geodaetica et Cartographica Sinica, vol. 39, no. 5, pp. 540-545, Oct. 2010.

[2] B. Yang, L. Fang, Q. Li, and J. Li, "Automated extraction of road markings from mobile lidar point clouds," Photogrammetric Engineering \& Remote Sensing, vol. 78, no. 4, pp. 331-338, Apr. 2012.

[3] D. Zhang, L. Li, and Y. Li, "Urban roads automated extracting from mobile laser scanning point clouds," Bulletin of Surveying and Mapping, no.7, pp. 30-34, Jul. 2016.

[4] M. Montemerlo, J. Becker, S. Bhat, H. Dahlkamp, and S. Ettinger, et al, "Junior: The stanford entry in the urban challenge," Journal of Field Robotics, vol. 25, no. 9, pp. 569-597, 2008.

[5] J. Byun, K. Na, B. Seo, and M. Roh, "Drivable road detection with 3D point clouds based on the MRF for intelligent vehicle," Springer Tracts in Advanced Robotics, vol. 105, pp. 49-60, Jan. 2015.

[6] Z. Zhu and J. L. Liu, "Real-time Markov random field based ground segmentation of 3D Lidar data," Journal of Zhejiang University (Engineering Science), vol. 49, no. 3, pp. 464-469, Mar. 2015.

[7] S. Kammel and B. Pitzer, "Lidar-based Lane Marker Detection and Mapping," 2008 IEEE Intelligent 
Vehicles Symposium, Eindhoven, Netherlands, Jun. 2008, pp. 1137-1142.

[8] F. Moosmann, O. Pink, and C. Stiller, "Segmentation of 3D Lidar Data in Non-Flat Urban Environments Using a Local Convexity Criterion," 2009 IEEE Intelligent Vehicles Symposium, Xi'an, China, Jul. 2009, pp. 215220.

[9] M. Himmelsbach, F. V. Hundelshausen, and H. J. Wuensche, "Fast segmentation of 3D point clouds for ground vehicles," 2010 IEEE Intelligent Vehicles Symposium, La Jolla, CA, USA, Aug. 2010, pp. 560565.

[10]P. Narksri, E. Takeuchi, Y. Ninomiya, Y. Morales, and N. Akai, et al, "A Slope-robust Cascaded Ground Segmentation in 3D Point Cloud for Autonomous Vehicles," 2018 21st International Conference on Intelligent Transportation Systems, Maui, HI, USA, Dec. 2018, pp. 497-504.

[11]D. Zermas, I. Izzat, and N. Papanikolopoulos, "Fast segmentation of 3D point clouds: A paradigm on LiDAR data for autonomous vehicle applications," 2017 IEEE International Conference on Robotics and Automation, Singapore, Jul. 2017, pp. 5067-5073.

[12]Z. Li, T. M. Wang, K. L. Hu, X. F. Li, and X. Wang, "Lidar object real-time detection method under complex environment", Laser Journal, vol. 39, no. 3, pp. 41-46, Mar. 2018.

[13] V. H. Le, H. Vu, T. T. Nguyen, T. L. Le, and T. H. Tran, "Acquiring qualified samples for RANSAC using geometrical constraints," Pattern Recognition Letters, vol. 102, pp. 58-66, Dec. 2017.

[14]N. Demir, "Automated detection of 3D roof planes from lidar data," Journal of the Indian Society of Remote Sensing, vol. 46, no. 8, pp. 1265-1272, Jul. 2018.

[15]M. Fischler and R. Bolles, "Random sample consensus: A paradigm for model fitting with applications to image analysis and automated cartography," Communications of the ACM, vol. 24, no. 6, pp. 381-395, Jun. 1981.

[16]R. Schnabel, R. Wahl, and R. Klein, "Efficient RANSAC for point-cloud shape detection," Computer Graphics Forum, vol. 26, no. 2, pp. 214-226, Jun. 2007.

[17]O. Chum and J. Matas, "Optimal randomized RANSAC," IEEE Transactions on Pattern Analysis and Machine Intelligence, vol. 30, no. 8, pp. 1472-1482, Jun. 2008.

[18]R.Y. Wei, X. G. Ruan, N.G. Yu, J. Huang, and X. Q. Zhu, et al, "Method of sample consensus based on Skinner operant conditioning," Control and Decision, vol. 30, no. 2, pp. 235-240, Oct. 2014.
[19]S. Lu, Y.J. Lei, W.W. Kong, and Y. Lei, "Fundamental matrix estimation based on probability analysis and sampling consensus," Control and Decision, vol. 27, no. 3, pp. 425-430, Mar. 2012.

[20]K. Wang, S. M. Jia, and X. Z. Li, "Improved RANSAC algorithm based on total probability updating," Control and Decision, vol. 32, no. 3, pp. 427-434, Jan. 2017.

[21] K. Wada, M. Murooka, K. Okada, and M. Inaba, "3D Object Segmentation for Shelf Bin Picking by Humanoid with Deep Learning and Occupancy Voxel Grid Map," 2016 IEEE-RAS International Conference on Humanoid Robots, Cancun, Mexico, Jan. 2016, pp. 1149-1154.

[22]M. F. Zhang, R. Fu, Y. S. Guo, Y. Q. Shi, W. D. Cheng, "Road segmentation method based on irregular three dimensional point cloud," Journal of Jilin University, vol. 47, no. 5, pp. 1387-1394, Mar. 2017.

Yawei Zhao, born in Xinmin, Liaoning, China, in April 1976. The author graduated from Shenyang University, China with a bachelor's degree in Industrial Automation in July 1999. She graduated from Northeastern University, China with a master's degree in Electrical Engineering Theory and New Technology in March 2006. Now She is mainly engaged in the research of electronic measurement technology, intelligent detection and control system.

Since July 2006, she has worked as a lecturer at Shenyang Ligong University, China.

\section{Contribution of individual authors to the creation of a scientific article (ghostwriting policy)}

Yawei Zhao performed algorithm design, programming implementation, and designed the overall experimental plan.

Yanju Liu was responsible for the preprocessing of the raw data of the lidar and the analysis of the experimental data.

Yang $\mathrm{Yu}$ was responsible for building a simulation environment based on Gazeb.

Jiawei Zhou is responsible for the communication between the lidar and the industrial computer.

\section{Creative Commons Attribution License 4.0 (Attribution 4.0 International, CC BY 4.0)}

This article is published under the terms of the Creative Commons Attribution License 4.0

https://creativecommons.org/licenses/by/4.0/deed.en_US 